\title{
DIFFUSION BY CONTINUOUS MOVEMENTS
}

\author{
$B y$ G. I. TaYLoR.
}

[Received Mray 22nd, 1920.-Read June 10th, 1920.]

\section{Introduction.}

It has been shown by the author, ${ }^{*}$ and others, that turbulent motion is capable of diffusing heat and otber diffusible properties through the interior of a fluid in much the same way that molecular agitation gives rise to molecular diffusion. In the case of molecular diffusion the relationship between the rate of diffusion and the molecular constants is known; a large part of the Kinetic Theory of Gases is devoted to this question. On the other hand, nothing appears to be known regarding the relationship between the constants which might be used to determine any particular type of turbulent motion and its "diffusing power."

The propositions set down in the following pages are the result of efforts to solve this problem.

In order to simplify matters as much as possible the transmission of heat in one direction only, that of the axis of $x$, will be considered. We shall take the case of an incompressible fluid whose temperature $\theta$, at time $t=0$, depends only on $x$, and increases or decreases uniformly with $x$. Initially therefore $\partial \theta / \partial x$ is constant and equal to $\beta$, say.

Now suppose that the fluid is moving in turbulent motion, so that the distribution of temperature is continually altering. Suppose that the turbulent motion could be defined by means of the Lagrangien equations of fluid motion, so that the coordinates $(x, y, z)$ of a particle are given in terms of its initial coordinates $(a, b, c)$ at the time $t=0$, and of $t$.

Since the temperature of any particle is supposed to remain constant during the motion, the temperature at the point $(x, y, z)$ at time $t$, which will be represented by the symbol $\theta(x, y, z)$ is $\theta(a, 0)$, which represents the temperature at $x=a$ at time $t=0$.

" "Eddy Motion in the Atmosphere," Plicl. Trans., 1915, p. 1. 
Since the rate of increase in temperature with $x$ is constant when $t=0$,

$$
\theta(a, 0)=\theta(x, 0)-(x-a) \beta .
$$

The average rate at which heat is being conveyed across unit area of a plane perpendicular to the axis of $x$ is evidently equal to $-\rho \sigma \beta$ multiplied by the average value of $u(x-a)$ over a large area of a plane perpendicular to the axis of $x$. In these expressions $u$ represents the velocity of a particle of fluid in the direction of the axis of $x, \rho$ is the density, and $\sigma$ the specific heat, so that $\rho \sigma$ is the heat capacity of unit volume of the fluid.

No doubt the average value of $u(x-a)$, which must be obtained from considerations of the particular nature of the turbulent motion in question, depends on the mean motion of the fluid ; but if experimental data exist, as in fact they do, which enable its value to be calculated, it is of interest to enquire what types of turbulent motion are capable of producing the observed distribution of temperature.

In order to simplify matters still further it will be assumed that the turbulent motion is uniformly distributed throughout space. The mean value of $u(x-a)$ will then be the sume for every layer and will be equal to the mean value throughout space. This quantity will be expressed by the symbol $[u(x-a)]$.

Owing to the fact that the fluid is incompressible $[u(x-a)]$ could be calculated either by taking a rectangular element $\delta x \delta y \delta z$, at time $t$, finding the corresponding value of $u(x-a)$ and integrating throughout space; or by taking an element $\delta a \delta b \delta c$ at time $t=0$, finding the corresponding value of $u(x-a)$ at time $t$, and integrating. The second method will be adopted.

Fixing our attention on a particle of fluid, it will be noticed that

$$
u=\frac{\partial x}{\partial t} \text { and } x-a=\int_{0}^{t} u d t .
$$

Hence, writing $X$ for $x-a$,

$$
[u(x-a)]=\left[X \frac{d X}{d t}\right]=\frac{1}{2}\left[\frac{d X^{2}}{d t}\right]=\frac{1}{2} \frac{d}{d t}\left[X^{2}\right] .
$$

In this ideally simplified system therefore the rate at which heat is transferred in the direction of the axis of $x$ is determined by the rate of increase of the mean value of the square of the distance, parallel to the axis of $x$, which is moved through by a particle of fluid in time $t$.

If a physicist were to try to define the characteristic features of any particular case of turbulent motion, with a view to discussing statistically 
its effect as a conductor of heat, he would probably first fix his attention on the mean energy of the motion. That is to say, he would determine $\left[u^{2}\right]$.

$\mathrm{He}$ would then perhaps notice that it is not sufficient to determine $\left[u^{2}\right]$. With a given value of $\left[u^{2}\right]$ it is possible for the turbulent motion to be associated with a small or a large transfer of heat, according to whether a particle frequently, or infrequently, reverses its direction of motion. It would therefore be necessary to detine some characteristic of the motion which differentiates between the cases in which the changes in the velocity of a particle are rapid, and those in which they are slow. A suitable characteristic to choose would be $\left[\left(\frac{d u}{d t}\right)^{2}\right]$.

Further investigation would show that it is necessary also to define

$$
\left[\left(\frac{d^{2} u}{d t^{2}}\right)^{2}\right], \ldots,\left[\left(\frac{d^{n} u}{d t^{n}}\right)^{2}\right], \ldots
$$

The relationship between

$$
\frac{1}{2} \frac{d}{d t}\left[X^{2}\right] \text { and }\left[u^{2}\right], \quad\left[\left(\frac{d u}{d t}\right)^{2}\right], \ldots,\left[\left(\frac{d^{n} u}{d t^{n}}\right)^{2}\right], \ldots
$$

is discussed in the following pages. The problem is in some respects similar to that known as "The drunkard's walk," or to Karl Pearson's* problem of the random migration of insects, when the motion is limited to one dimension; but in the course of the investigation some curious propositions have come to light concerning the mean values of continuously varying quantities which may perhaps be of interest to mathematicians, as well as to physicists.

In the course of the work no discussion of the convergency of the series used is attempted. The work must therefore be regarded as incomplete. The author feels that such questions might be examined with advantage by a pure mathematician, and it is in the hope of interesting one of them that he wishes to offer this paper to the London Mathematical Society.

\section{Discontinuous Motion.}

Before proceeding to consider the continuous version of the problem of random migration in one dimension, the discontinuous case will be

* Drapers' Company Memoirs. 
extended slightly, so as to make it bear some resemblance to the continuous case.

Suppose that a point starts moving with uniform velocity $v$ along a line, and that after a time $\tau$ it suddenly makes a fresh start and either continues moving forward with velocity $v$ or reverses its direction and moves back over the same path with the same velocity $v$. Suppose that this process is repeated $n$ times and that we consider the mean values of the quantity concerned for a very large number of such paths.

Let $x_{r}$ be the distance moved over in the $r$-th interval. Then $x_{r}$ is numerically equal to $v \tau$, but its sign may be either positive or negative and each occurs an equal number of times in considering the average. If $X_{n}$ is the standard deviation or "root mean square" of the distance moved by the point from the original position after time $n \tau$, then

$$
X_{n}^{2}=\left[\left(x_{1}+x_{2}+x_{3}+\ldots+x_{n}\right)^{2}\right],
$$

where the square bracket indicates that the mean value is taken for all the paths.

Hence $\quad X_{n}^{2}=n d^{2}+2\left[x_{1} x_{2}+x_{1} x_{3}+\ldots+x_{r} x_{s}+\ldots\right]$,

where

$$
d=v \tau \text {. }
$$

If there is no correlation between any two $x$ 's,

$$
\left[x_{r} x_{s}\right]=0 \text {. }
$$

Hence

$$
X_{n}^{2}=n d^{2}, \quad \text { or } \quad X_{n}=d \sqrt{ } n=v \sqrt{ }\left(\tau T_{n}\right),
$$

where $T_{n}$ is the total time during which the migration has been taking place. It will be seen therefore that $X_{n}$ is proportional to $\sqrt{ } T_{n}$.

Actually in a turbulent fluid or in any continuous motion there is necessarily a correlation between the movement in any one short interval of time and the next. This correlation will evidently increase as the interval of time diminishes, till, when the time is short compared with the time during which a finite change in velocity takes place, the coefficient of correlation tends to the limiting value unity.

This idea will now be introduced into equation (1).

To begin with let us make the arbitrary assumption that $x_{r}$ is correlated with $x_{r+1}$ by a correlation coefficient $c$. Suppose also that the partial correlations of $x_{r}$ with $x_{r+2}, x_{r+3}, \ldots$ are all zero. The correlation coefficient between $x_{r}$ and $x_{r+2}$ is then $c^{2}$. Between $x_{r}$ and $x_{r+s}$ it is $c^{s}$.

The value of $2\left[x_{1} x_{2}+x_{1} x_{3}+\ldots+x_{r} x_{8}+\ldots\right]$ is then

$$
2 d^{2}\left\{n c+(n-1) c^{2}+(n-2) c^{8}+\ldots+c^{n}\right\} .
$$


The series in the \{\} bracket is easily summed. Substituting its value in (1) it will be found that

$$
X_{n}^{2}=d^{2}\left\{n+\frac{2 n c}{1-c}-\frac{2 c^{2}\left(1-c^{n}\right)}{(1-c)^{2}}\right\},
$$

or, putting $n=T_{n} / \tau$, and $d=v \tau$,

$$
X_{n}^{2}=v^{2}\left\{\left(\frac{1+c}{1-c} \tau\right) T_{n}-\frac{2 c^{2}\left(1-c^{n}\right) \tau^{2}}{(1-c)^{2}}\right\} .
$$

By reducing $\tau$ indefinitely we can evidently make the case approximate to some sort of continuous migration, but in order that $X_{n}, v$ and $T_{n}$ may be finite and tend to a definite limit as $\tau$ is decreased, it is necessary that $\left(\frac{1+c}{1-c}\right) \tau$ and $\frac{2 c^{2}\left(1-c^{n}\right) \tau^{2}}{(1-c)^{2}}$ must also tend to a definite limit. That is to say, $1-c$ must be proportional to $\tau$.

Let $\frac{\tau}{1-c}$ tend to the limit $A$ when $\tau$ and $1-c$ tend to zero.

Then $X_{n}^{2}$ tends to the limiting value

$$
v^{2}\left\{2 A T_{n}-2 A^{2}\left(1-e^{-T_{n} / A}\right)\right\},
$$

or, dropping the suffixes which are no longer necessary,

$$
\sqrt{ }\left[X^{2}\right]=v \sqrt{ }\left\{2 A T-2 A^{2}\left(1-e^{-T / A}\right)\right\},
$$

where $X$ is the distance traversed by a particle during a flight extending over an interval of time $T$, and the "root mean square" is taken for a large number of such flights.

When $T$ is small this reduces to $\sqrt{ }\left[X^{2}\right]=v T$, which is exactly what we should expect when the time is so short that the correlation coefficient $c^{n}$, between the first and last small element of migration has not fallen appreciably away from unity.

When $T$ is large $\sqrt{ }\left[X^{2}\right]=v \sqrt{ }(2 A T)$, so that the amount of "diffusion" is proportional to the square root of the time. The constant $A$ evidently measures the rate at which the correlation coefficient between the direction of an infinitesimal path in the migration and that of an infinitesimal path at a time $T$, say, later, fulls off with increasing values of $T$.

We have now seen how it is possible by introducing the idea of a correlation between the directions of the successive jumps in a random migration, to keep the standard deviation of the distance of migration constant, no matter how small the infinitesimal paths of the migration may be.

The migration is still a discontinuous one however. It suffers also 
from the disadvantage of depending on a special assumption, numely, that there is a definite correlation between the direction of motion in one infinitesimal element of path, and that in its immediate neighbours, but that there is no partial correlation between the directions of motion in paths which are not neighbours. This means that there is a special law of correlation between the directions of the paths at finite intervals of time. The correlation coefficient between the direction of an infinitesimal path and that of the path which occurs at a time $T=n \tau$ later, is evidently $c^{n}$. This may be written

$$
\{1-(1-c)\}^{n}=(1-\tau / A)^{n}=(1-\tau / A)^{T / \tau} .
$$

When $\tau$ is small this tends to the limit $e^{-T / A}$.

We are therefore limiting ourselves to the particular type of motion in which the direction of an infinitesimal path is correlated to that at time $T$ later by the correlation coefficient* $e^{-T / A}$

\section{Diffusion by continuous Movements.}

The work just described, though not particularly useful for our present purpose, is useful in that it gives rise to ideas about how problems of migration or diffusion by continuous movements may be treated. In what follows these ideas are worked out and the conditions of motion which determine the laws of diffusion are found.

Before proceeding to discuss diffusion, however, it will be necessary to prove a few statistical properties of continuously varying quantities.

Suppose that we wish to express the characteristic properties of the variations of some quantity which varies continuously, but which appears to have no very definite law of variation. Suppose, for instance, it is desired to define the characteristic features of a barograph record. There are no obvious periods, nor is there any definite constant amplitude of variation in barometric pressure, yet there are certain properties of the curve which can be defined. If we take the standard deviation of pressure from its mean value during a year, it will be found to be practically constant from year to year. If $p$ represents the deviation from the mean pressure, this standard deviation is $\sqrt{ }\left[p^{2}\right]$, where the square bracket now indicates that the mean value of $p^{2}$ has been taken over a long period of

* Incidentally it will be noticed that the correlation between the direction of motion at one instant and that at time $t$ earlier is also $e^{-T / A}$. It is obvious that we cannot consider the value of the expression $e^{-T / A}$ when $T$ is negative. 
time. One property of the curve which we can define, therefore, is the constancy of $\sqrt{ }\left[p^{2}\right]$ during successive long periods.

The statistical properties of the barograph curve are by no means. completely determined by this. It is possible to imagine an infinite variety of barograph curves with a given standard deviation of $p$. They might, for instance, have a large number of peaks in the curve during a given interval of time or a small number. In the former case the standard deviation of $d p / d t$ might be expected to be larger than in the latter. We can, therefore, define the curve still further by specifying the standard deviations of $d p / d t$.

It appears that, from a given barograph curve, it is theoretically possible to find the standard deviations of $p, d p / d t, d^{2} p / d t^{2}, \ldots, d^{n} p / d t^{n}, \ldots$. Let us assume that all these are constant from year to year.

Now suppose that we begin by specifying certain arbitrary standard deviations for $p, d p / d t$, \&c., and that we try to construct a possible barograph curve from them. We are at once brought up against a difficulty. Suppose that we have specified a large number for the standard deviation of $d p / d t$, i.e. $\sqrt{ }\left[(d p / d t)^{2}\right]$ and small numbers for $\sqrt{ }\left[p^{2}\right]$ and $\sqrt{ }\left[\left(d^{2} p ! d t^{2}\right)^{2}\right]$. It is evident that if we begin constructing the curve with a large value of $d p / d t$ at a point where $p=0$, the fact that the value of $\sqrt{ }\left[\left(d^{2} p / d t^{2}\right)\right]$ is small means that it will be a long time before $d p / d t$ changes sign. Hence it will be a long time before $p$ attains its maximum value, and during that time $p$ must have attained a large value. Hence, if the standard deviation of $d p / d t$ is large and that of $d^{2} p / d t^{2}$ is small, the standard deviation of $p$ must be large. It is evident therefore that there must be some relation. ships between the standard deviations and the curve of which we have not yet taken account. We shall now see what these are.

Suppose that we observe the values $p_{1}, p_{2}, p_{3}, \ldots, p_{n}$ of $p$ at a large number of successive times $t_{1}, t_{2}, t_{3}, \ldots, t_{n}$. Suppose further that we observe the values $p_{1}+\delta p_{1}, p_{2}+\delta p_{2}, p_{3}+\delta p_{3}, \ldots, p_{n}+\delta p_{n}$, at times. $\dot{t}_{1}+\delta t, t_{2}+\delta t, t_{3}+\delta t, \ldots, t_{n}+\delta t$, where $\delta t$ is a small interval of time. Then, if $t_{1}, t_{2}, \ldots, t_{n}$ are taken at random

$$
\left[p^{2}\right]=\left(p_{1}^{2}+p_{2}^{2}+\ldots+p_{n}^{2}\right) / n,
$$

and since we are considering a curve in which $\left[p^{2}\right]$ is constant, $\left[p^{2}\right]$ is also, to the first order, equal to

$$
\begin{aligned}
& \frac{1}{n}\left\{\left(p_{1}+\frac{d p_{1}}{d t} \delta t\right)^{2}+\left(p_{2}+\frac{d p_{2}}{d t} \delta t\right)^{2}+\ldots+\left(p_{n}+\frac{d p_{n}}{d t} \delta t\right)^{2}\right\} \\
= & {\left[p^{2}\right]+2\left[p \frac{d p}{d t}\right] \delta t . }
\end{aligned}
$$


It appears, therefore, that we can differentiate the quantities inside square brackets which indicate a mean value.

Hence the condition that $\left[p^{2}\right]$ shall be a constant is

$$
\left[p \frac{d p}{d t}\right]=0 .
$$

There is, therefore, no correlation between $p$ and $d p / d t$.

Now differentiate (5) once more,

$$
\left[p \frac{d^{2} p}{d t^{2}}\right]+\left[\left(\frac{d p}{d t}\right)^{2}\right]=0 .
$$

Hence by the definition of a correlation coefficient, there is a negative correlation between $p$ and $d^{2} p / d t^{2}$ equal to

$$
\nu=-\frac{\left[\left(\frac{d p}{d t}\right)^{2}\right]}{\sqrt{ }\left[p^{2}\right] \sqrt{ }\left[\left(\frac{d^{2} p}{d t^{2}}\right)^{2}\right]} .
$$

A consequence of the existence of this correlation coefficient $\nu$ is evidently. that $\left[(d p / d t)^{2}\right]$ cannot be greater than $\sqrt{ }\left[p^{2}\right] \sqrt{ }\left[\left(d^{2} p / d t^{3}\right)^{2}\right]$, a statement. which agrees with the remarks above.

The way in which the correlation coefficient affects the characteristifeatures of the $p, t$ curve is easily seen. Suppose it is large, i.e. nearly equal to -1 ; then the curve will look something like curve (a), Fig. 1 .

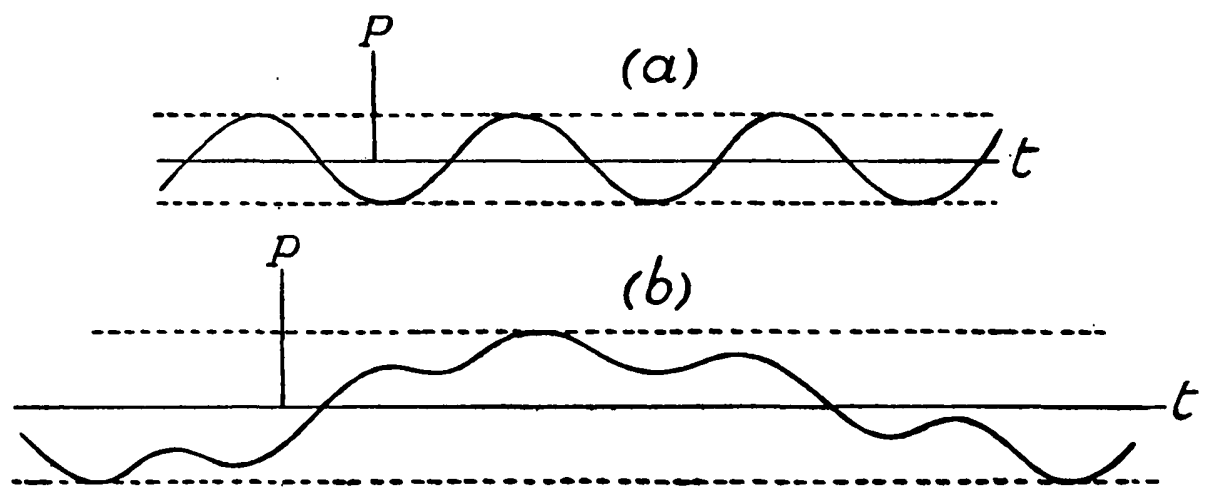

FIG. 1.

Suppose the correlation coefficient between $p$ and $d^{2} p / d t^{2}$ is small, but that the standard deviations of $d p / d t$ and $d^{2} p / d t^{2}$ are the same as in curve (a), Fig. 1, then the slopes and curvatures will be of the same magnitude 
as in curve (a), but the curvature will not always be concave to the mean line. This is shown in curve (b), Fig. 1.

It is evident that the standard deviation of $p$ is greater in $(b)$ than it is in (a). This is expressed by the formula (7), for if the standard devia. tions of $d p / d t$ and $d^{2} p / d t^{2}$ are fixed, then the standard deviation of $p$ is, according to (7), inversely proportional to $\nu$.

Since the staudard deviation of $d p / d t$ has also been given as constant it can be treated exactly in the same way as the standard deviation of $p$, thus differentiating $\left[(d p / d t)^{2}\right]$, we have

$$
\left[\frac{d p}{d t} \frac{d^{2} p}{d t^{2}}\right]=0
$$

and differentiating this again

$$
\left[\frac{d p}{d t} \frac{d^{3} p}{d t^{3}}\right]+\left[\left(\frac{d^{2} p}{d t^{2}}\right)^{2}\right]=0
$$

But differentiating (6) again

$$
\left[p \frac{d^{3} p}{d t^{3}}\right]+3\left[\frac{d p}{d t} \frac{d^{2} p}{d t^{2}}\right]=0
$$

Hence, from (8),

$$
\left[p \frac{d^{3} p}{d t^{3}}\right]=0 \text {. }
$$

Differentiating (10), $\quad\left[p \frac{d^{4} p}{d t^{4}}\right]+\left[\frac{d p}{d t} \frac{d^{3} p}{d t^{3}}\right]=0$.

Hence, from (9), $\quad\left[p \frac{d^{4} p}{d t^{4}}\right]-\left[\left(\frac{d^{2} p}{d t^{2}}\right)^{2}\right]=0$.

Proceeding in this way it can be shown that

and

$$
\begin{aligned}
{\left[p \frac{d^{2 n} p}{d t^{2 n}}\right] } & =(-1)^{n}\left[\left(\frac{d^{n} p}{d t^{n}}\right)^{2}\right], \\
{\left[p \frac{d^{2 n+1} p}{d t^{2 n+1}}\right] } & =0 .
\end{aligned}
$$

The correlations to which $p$ and its differential coefficients must be subject in order that their standard deviations may be constant, have now been established. We can, therefore, now use these standard deviations to define some statistical properties of the curve.

In analysing any actual curve, it may be very difficult and tedious to obtain these standard deviations. There is, however, another method of defining the statistical properties of the curve which is equivalent to that 
given above, but which is likely to be much more manageable in practice. This method will now be considered.

Suppose that we take, as before, the values $p_{1}, p_{2}, p_{3}, \ldots, p_{n}$, of $p$ at a large number of times $t_{1}, t_{2}, t_{3}, \ldots, t_{n}$, chosen at random. Let us correlate them with the values $p_{1}^{\prime}, p_{2}^{\prime}, \ldots, p_{n}^{\prime}$, of $p$ at times $t_{1}+\xi, t_{2}+\xi, \ldots, t_{n}+\xi$, where $\xi$ is a finite interval of time which may be positive or negative. Let the coefficient of correlation so found be $R_{\xi}$. Then $R_{\xi}$ must evidently be a function of $\xi$.

If $p_{t}$ be the value of $p$ at time $t$, and $p_{t+\xi}$ be the value of $p$ at time $t+\xi$, then by definition

$$
\left[p_{t} p_{t+\xi}\right]=R_{\xi} \sqrt{ }\left[p_{t}^{2}\right] \sqrt{ }\left[p_{t+\xi}^{2}\right] ;
$$

but by hypothesis the standard deviation of $p$ does not vary, hence

and

$$
\left[p_{t}^{2}\right]=\left[p^{2}\right]=\left[p_{t+\xi}^{2}\right]
$$

$$
R_{\xi}=\left[p_{t} p_{t+\xi}\right] /\left[p^{2}\right] \text {. }
$$

Now expand $p_{t+\xi}$ in powers of $\xi$,

$$
p_{t+\xi}=p_{t}+\xi \frac{d p}{d t}+\frac{\xi^{2}}{2 !} \frac{d^{2} p}{d t^{2}}+\ldots
$$

Hence

$$
\left[p_{t} p_{t+\xi}\right]=\left[p_{t}^{2}\right]+\xi\left[p \frac{d p}{d t}\right]+\frac{\xi^{2}}{2 !}\left[p \frac{d^{2} p}{d t^{2}}\right]+-_{3 !}^{\xi^{3}}\left[p \frac{d^{3} p}{d t^{3}}\right] .
$$

Substituting for $\left[p \frac{d^{n} p}{d t^{n}}\right]$ from (11), (13) becomes

$$
\left[p_{t} p_{t+\xi}\right\rfloor=\left[p^{2}\right]+\xi(0)-\frac{\xi^{2}}{2 !}\left[\left(\frac{d p}{d t}\right)^{2}\right]+\frac{\xi^{3}}{3 !}(0)+\frac{\xi^{4}}{4 !}\left[\left(\frac{d^{2} p}{d t^{2}}\right)^{2}\right] \ldots
$$

Hence, from (12),

$$
R_{\xi}=1-\frac{\xi^{2}}{2 !} \frac{\left[\left(\frac{d p}{d \cdot t}\right)^{2}\right]}{\left[p^{2}\right]}+\frac{\xi^{4}}{4 !} \frac{\left[\left(\frac{d^{2} p}{d t^{2}}\right)^{2}\right]}{\left[p^{2}\right]}-\ldots+(-1)^{n} \frac{\xi^{2 n}}{2 n !} \frac{\left[\left(\frac{d^{n} p}{d t^{n}}\right)^{2}\right]}{\left[p^{2}\right]} .
$$

It will be seen that, as might have been expected, $R_{\xi}$ is an even function of $\xi$.

As an example of the method let us take the case where it is known that $p=\sin (t+\epsilon)$, where $\epsilon$ may take all possible values, all of which are equally probable. In this case

$$
\left[p^{2}\right]=\frac{1}{2}, \quad\left[(d p / d t)^{2}\right]=\frac{1}{2}, \quad \ldots, \quad\left[\left(d^{n} p / d t^{n}\right)^{2}\right]=\frac{1}{2}, \quad \ldots,
$$


(14) therefore becomes

$$
R_{\xi}=1-\frac{\xi^{2}}{2 !} \frac{\left(\frac{1}{2}\right)}{\left(\frac{1}{2}\right)}+\frac{\xi^{4}}{4 !} \frac{\left(\frac{1}{2}\right)}{\left(\frac{1}{2}\right)}-\ldots
$$

This is the series for $\cos \xi$. Hence $R_{\xi}=\cos \xi$. The correlation between the value of $p$ at any time and its value when $t$ is increased by any odd multiple of $\frac{1}{2} \pi$ is 0 . This is obviously true since there is no correlation between $\sin (t+\epsilon)$ and $\sin \left\{t+\epsilon+(2 n+1)\left(\frac{1}{2} \pi\right)\right\}$ as $\epsilon$ varies.

The correlations between $p$ and its differential coefficients given in (11) are evidently also true.

\section{Application to Diffusion by continuous Movements.}

The theorems which have just been proved will now be used to find out what are the essential properties of the motion of a turbulent fluid which makes it capable of diffusing through the fluid properties such as temperature, smoke content, colouring matter or other properties which adhere to each particle of the fluid during its motion.

Consider a condition in which the turbulence in a fluid is uniformly distributed so that the average conditions of every point in the fluid are the same. Let $u$ be the velocity parallel to a fixed direction, which we will call the axis of $x$, of the particle on which our attention is fixed. It will now be shown that the statistical properties which were defined above (now in relation to $u$ instead of $p$ ) are sufficient to determine the law of diffusion, i.e. the law which governs the average distribution of particles initially concentrated at one point, at any subsequent time.

Suppose that the statistical properties of $u$ are known in the form given above, that is to say, suppose that $\left[u^{2}\right]$ and $R_{\xi}$ are known. $R_{\xi}$ is now the correlation coefficient between the value of $u$ for a particle at any instant, and the value of $u$ for the same particle after an interval of time $\xi$.

Let $u_{t}$ represent the value of $u$ at time $t$. Consider the value of the definite integral

$$
\int_{0}^{t}\left[u_{t} u_{\xi}\right] d \xi .
$$

By the definition of $R_{\xi}$ this is equal to

$$
\left[u_{t}^{2}\right] \int_{0}^{t} R_{\xi-t} d \xi .
$$


Hence, since $\left[u^{2}\right]$ does not vary with $t$, and $R_{\xi}$ is an even function of $\xi$,

$$
\int_{0}^{t}\left[u_{t} u_{\xi}\right] d \xi=\left[u^{2}\right] \int_{0}^{t} R_{\xi} d \xi .
$$

Evidently one can integrate inside the square bracket just as one can differentiate. Hence

$$
\int_{0}^{t}\left[u_{t} u_{\xi}\right] d \xi=\left[u_{t} \int_{0}^{t} u_{\xi} d \xi\right]=\left[u_{t} X\right],
$$

or, in the notation of the introduction, $[u X]$.

Hence

$$
\begin{aligned}
{\left[u^{2}\right] \int_{0}^{t} R_{\xi} d \xi } & =[u X] \\
& =\frac{1}{2} \frac{d}{d t}\left[X^{2}\right]
\end{aligned}
$$

and

$$
\left[X^{2}\right]=2\left[\iota^{2}\right] \int_{0}^{T} \int_{0}^{t} R_{\xi} d \xi d t,
$$

where $X$ is the distance traversed by a particle in time $T$.

Equation (18) is rather remarkable because it reduces the problem of diffusion, in a simplified type of turbulent motion, to the consideration of a single quantity, namely, the correlation coefficient between the velocity of a particle at one instant and that at a time $\xi$ later.

Let us now consider the physical meaning of (18), when $T$ is so small that $R_{\xi}$ does not differ appreciably from 1 during the interval $T$. In this case

$$
\int_{0}^{T} \int_{0}^{l} R_{\xi} d \xi d t=\frac{1}{2} T^{2}
$$

so that (18) becomes

$$
\begin{aligned}
{\left[X^{2}\right] } & =\left[u^{2}\right] T^{2}, \\
\sqrt{ }\left[X^{2}\right] & =T \sqrt{ }\left[u^{2}\right] .
\end{aligned}
$$

That is to say, the standard deviation of a particle from its initial position is proportional to $T$ when $T$ is small. This is what we should expect provided the time $T$ is so small that the velocity does not alter appreciably while the particle is moving over the path.

Now consider how one would anticipate that $R_{\xi}$ would vary with $\xi$ in a turbulent fluid. The most natural assumption seems to be that $R_{\xi}$ would fall to zero for large values of $\xi$. It might remain positive as in 
the curve shown in Fig. 2, or it might become negative or oscillate before

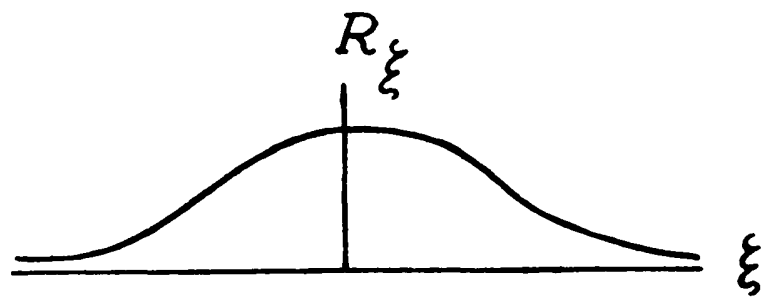

Fia. 2.

falling off to zero. In either case it seems probable that it will be possible to define an interval of time $T_{1}$, such that the velocity of the particle at the end of the interval $T_{1}$ has no correlation with the velocity at the beginning. In this case suppose that $\lim _{t \rightarrow \infty} \int_{0}^{t} R_{\xi} d \xi$ is finite and equal to $I$. Then at time $T\left(>T_{1}\right)$ after the beginning of the motion

$$
\frac{d}{d t}\left[X^{2}\right]=2\left[u^{2}\right] I,
$$

so that $\left[X^{2}\right]$ increases at a uniform rate. In the limit when $\left[X^{2}\right]$ is large

$$
\sqrt{ }\left[X^{2}\right]=\sqrt{ }\left(2 I T\left[u^{2}\right]\right),
$$

so that the standard deviation of $X$ is proportional to the square root of the time.

This, therefore, is a property which a continuous eddying motion may be expected to have which is exactly analogous to the properties of discontinuous random migration in one dimension.

It will be noticed that when $T>T_{1}$,

$$
[X u]=\left[u^{2}\right] 1 \text {. }
$$

Hence $[X u]$ is constant in spite of the fact that $\left[X^{2}\right]$ continually increases. In order that this may be the case $X$ must always be positively correlated with $u$, but the correlation coefficient must decrease with in. creasing $\left[X^{2}\right]$. If $v_{X u}$ represents the correlation coefficient between $X$ and $u$

$$
v_{X u}=-\frac{[X u]}{\sqrt{ }\left[X^{2}\right] \sqrt{ }\left[u^{2}\right]} \doteq \frac{I \sqrt{ }\left[u^{2}\right]}{\sqrt{ }\left[X^{2}\right]},
$$

and in the limit when $T \rightarrow \infty$,

$$
\nu_{\lambda u}=\frac{I \sqrt{ }\left[u^{2}\right]}{\sqrt{ }\left(21 T\left[u^{2}\right]\right)}=\sqrt{ } \frac{I}{2 T} .
$$


It is interesting to compare the expression (18) for $\left[X^{2}\right]$ with the expression given in (3) for the standard deviation of $X$ in the special case of discontinuous motion considered thəre.

In that case $R_{\xi}$ was shown in (4) to be $e^{-\xi / A}$ In the continuous case if we write $R_{\xi}=e^{-\xi / 4}$, (21) becomes

$$
\begin{aligned}
{\left[X^{2}\right] } & =2\left[u^{2}\right] \int_{0}^{T} \int_{0}^{t} e^{-\xi / A} d \hat{\xi} d t \\
& =2\left[u^{2}\right] \int_{0}^{T} A\left(1-e^{-t / A}\right) d t \\
& =2\left[u^{2}\right]\left\{A T-A^{2}\left(1-e^{-T / A}\right) !\right.
\end{aligned}
$$

In the discontinuous case it was shown in (3) that

$$
\sqrt{ }\left[X^{2}\right]=v \sqrt{ }\left\{2 A T-2 A^{2}\left(1-e^{-T / A}\right)\right\} .
$$

It is evident that this is exactly the same as (23) except that $\sqrt{ }\left[u^{2}\right]$ has been substituted for the constant $v$ which occurred in the discontinuous case.

If as a result of experiments on diffusion, it were possible to obtain a curve representing $\left[X^{2}\right]$ as a function of $T$, it would be possible to use (18) as a means of discovering something about the nature of the turbulence, for (18) could be written

$$
\frac{\dot{d}^{2}}{d t^{2}}\left[X^{2}\right]=2\left[u u^{2}\right] R_{\xi},
$$

and $R_{\xi}$ could therefore be found.

In a recent communication to the Royal Society, ${ }^{*}$ Mr. L. F. Richardson has described some experiments on the diffusion of smoke emitted from a fixed point in a wind. Similar observations have been made on the smoke from factory chimneys by Mr. Gordon Dobson. $\dagger$ Both these observers came to the conclusion that, at small distances from the origin of the smoke, the surface containing the standard deviations of the smoke from a horizontal straight line to leeward of the source, is a cone. If the mean velocity of the wind is assumed to be uniform, the standard deviation in a short interval of time is therefore proportional to the time. At greater distances their observations indicate that this surface becomes like a paraboloid, so that the deviation of the smoke is proportional to the snuare root of the time.

* Phil. Trans., A, Vol. 221, p. 1.

† Advisory Committee for Aeronautics (Reports, 1919).

SER. 2 . VOL. 20 . NO. 1390 . 
Both these observational data are in agreement with equations (19) and (20).

Mr. Richardson's method consisted in taking a photograph of the smoke leaving a source and drifting down-wind. The exposure was not instantaneous, but extended over such a long period that a kind of composite photogragh was obtained showing the outer limits of the region containing the smoke. The general shape of the outline of this region is shown in Figs. 4 and 5 ; it is, as has been explained, a parabola with a pointed vertex. In some cases the paraboloidal part of the surface joined straight on to the conical part, as shown in Fig. 4, but in other cases there was a sort of neck between them as shown in Fig. 5. According to the theory set forth above this neck would be anticipated in cases where the $R_{\xi}$ curve contained negative values as shown in Fig. 3. An $R_{\xi}$ curve of this type might be due to some sort of regularity in the eddies of which the turbulent motion consists.

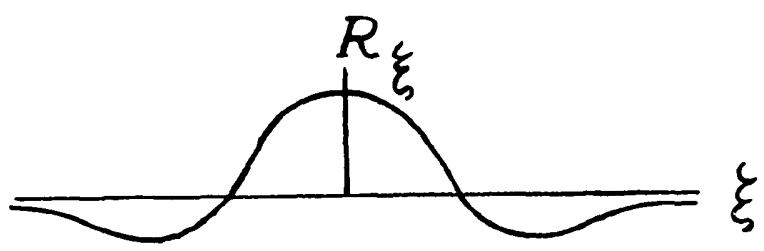

Fro. 3 .
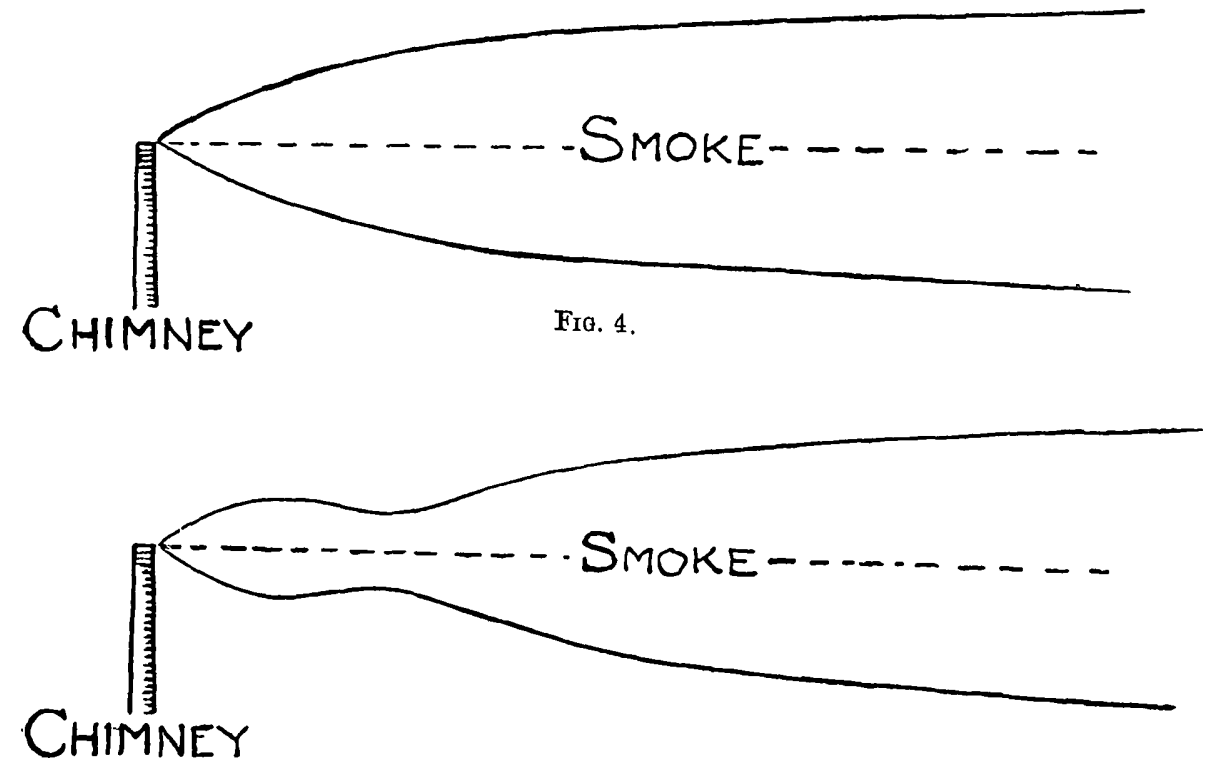

FIG. 5. 
It appears that both theory and observation indicate that $[X u]$ becomes constant after a certain interval of time (which depends of course on the value of $\xi$ at which $\int_{0}^{\xi} R_{\xi} d \dot{\xi}$ kecomes practically constant with increasing values of $\xi$ ). This is a matter of considerable interest in the theory of the conduction of heat by means of turbulence, because it indicates a reason why the "diffusing power" of any type of turbulence appears to depend so little on the molecular conductivity and viscosity of the fluid.

After writing this paper I showed it to Mr. Richardson, who informed me that he had already noticed the relations (11), and at my request he sent me his proof which follows.

Note on a Theorem by Mr. G. I. Taylor on Curves which Oscillate Irregularly

By Lewis F. Richardson.

The theorem referred to is proved on the hypothesis that the standard deviations of $p, d p / d t, d^{2} p / d t^{2}, \ldots, d^{n} p / d t^{n}$ are constant over any long time. It also follows, as will now be shown, from the rather different hypotheses which may be stated thus:-

(i) No one of $p, d p / d t, d^{2} p / d t^{2}$ has a standard deviation less than a certain lower limit.

(ii) The instantaneous values ( $t$ being time) of $p, d p / d t, d^{2} p / d t^{2}, \ldots$, never exceed in numerical value a certain upper limit.

We might state simple numerical upper and lower limits. But as we are dealing with oscillations, it will be as well to take a hint from the properties of the sine curve. If $p=c \sin s p$, then $\left|d^{n} p / d t^{n}\right|$ is not greater than $c s^{n}$, and the standard deviation of $d^{n} p / d t^{n}$ is $\sqrt{ } \frac{1}{2} c s^{n}$.

For our irregular curve let us define $B$ and $r$ and $A$ so that $|p|<B$ and $\left|d^{n} p / d t^{n}\right|<B r^{n}$.

The standard deviation of $p$ is greater than $A$ and that of $d^{n} p / d t^{n}$ is greater than $A r^{n}$.

It is required to find

$$
\frac{1}{t_{2}-t_{1}} \int_{t_{1}}^{t_{2}} p \frac{d^{2 n} p}{d t^{2 n}} d t
$$


Integrate by parts, successively, so as to differentiate the $p$ and to integrate $d^{2 n} p / d t^{2 n}$ until they both coincide in $d^{n} p / d t^{n}$. For example, when $n=5$, the result is

$$
\begin{aligned}
& \frac{1}{t_{2}-t_{1}} \int_{t_{1}}^{t_{2}} p \frac{d^{10} p}{d t^{10}} d t \\
= & \frac{1}{t_{2}-t_{1}} \int_{t_{1}}^{t_{2}}\left[-\frac{d^{9} p}{d t^{9}} p+\frac{d^{8} p}{d t^{8}} \frac{d p}{d t}-\frac{d^{7} p}{d t^{7}} \frac{d^{2} p}{d t^{2}}+\frac{d^{6} p}{d t^{6}} \frac{d^{3} p}{d t^{3}}-\frac{d^{5} p}{d t^{5}} \frac{d^{4} p}{d t^{4}}\right] \\
+ & \frac{1}{t_{2}-t_{1}} \int_{t_{1}}^{t_{2}}\left(\frac{d^{5} p}{d t^{5}}\right)^{2} d t .
\end{aligned}
$$

The expression in square brackets is less than $5 B^{2} r^{9}$ however long the interval $\left(t_{2}-t_{1}\right)$ may be, while $\int_{t_{1}}^{t_{2}}\left(\frac{d^{5} p}{d t^{5}}\right)^{2} d t$ is greater than $\left(t_{2}-t_{1}\right) A^{2} r^{10}$, and so increases with the interval.

Thus when $\left(t_{2}-t_{1}\right)$ is large enough, the term in square brackets becomes negligible. Generalizing the example, and taking account of the changes of sign introduced by partial integration,

$$
\lim _{t_{2}-t_{1} \rightarrow \infty} \frac{1}{t_{2}-t_{1}} \int_{t_{1}}^{t_{2}} p \frac{d^{2 n} p}{d t^{2 n}} d t \text { is } \frac{(-1)^{n}}{t_{2}-t_{1}} \int_{t_{1}}^{t_{2}}\left(\frac{d^{n} p}{d t^{n}}\right)^{2} d t .
$$

If in place of $d^{2 n} p / d t^{2 n}$ in (5) we had had a coefficient of odd order, the partial integrations, when pursued so as to lead back again to the original form, would have produced an arrangement of signs such that like terms were added. So that

$$
\lim _{t_{2}-t_{1} \rightarrow \infty} \frac{1}{t_{2}-t_{1}} \int_{t_{1}}^{t_{3}} p \frac{d^{2 n+1} p}{d t^{2 n+1}} d t=0 .
$$

This depends on the hypothesis (2) only. Hypothesis (1) does not come in here. It was needed in proving (7). 